\title{
Design and trajectory tracking control of a piezoelectric nano-manipulator with actuator saturations
}

\author{
Pengbo Liu ${ }^{a}$, Peng Yan ${ }^{\mathrm{a}, \mathrm{b}, *}$, Hitay Özbay ${ }^{\mathrm{c}}$ \\ ${ }^{a}$ Key Laboratory of High-efficiency and Clean Mechanical Manufacturing, Ministry of Education, School of Mechanical Engineering, Shandong University, \\ Jinan 250061, China \\ ${ }^{\mathrm{b}}$ School of Automation Science and Electrical Engineering, Beihang University, Beijing 100191, China \\ ${ }^{\mathrm{c}}$ Department of Electrical E' Electronics Engineering, Bilkent University, Ankara 06800, Turkey
}

\section{A R T I C L E I N F O}

\section{Article history:}

Received 30 December 2016

Received in revised form 27 December 2017

Accepted 5 April 2018

\section{Keywords:}

Nano-manipulator

Trajectory tracking

Parallel internal-model

Anti-windup compensator

$\mathcal{H}_{\infty}$ optimization

\begin{abstract}
A B S T R A C T
This paper presents the design of an XYZ nano-manipulator as well as the model based high precision tracking control of the nano-manipulating system. Aiming at large range and high mechanical bandwidth, the proposed mechanical design employs compound bridge-type amplifiers to increase the workspace without significant drop of stiffness. To further improve the system tracking performance and avoid possible actuator saturations, a robust anti-windup tracking control architecture combining a parallel internal-model based controller and an anti-windup compensator is adopted for the trajectory tracking of the designed nano-manipulating system. As a theoretical extension on a recent result [17], we further investigate the robust stability condition of the closed-loop system and formulate the optimization design of the anti-windup compensators as a two block $\mathcal{H}_{\infty}$ optimization problem solvable with the Nehari approach. Real time control experiments demonstrate excellent tracking performance and saturation compensation capability with tracking precision error less than $0.28 \%$, which significantly outperforms relevant algorithms in the literature.
\end{abstract}

(c) 2018 Elsevier Ltd. All rights reserved.

\section{Introduction}

Flexure-based multi-axial (XYZ) manipulators play a critical role in many ultra high precision applications such as optical microscope systems, optical fiber alignment, biological cell manipulation where fast and accurate multi-dimensional motions are needed $[1,2]$. Due to the nature of the nano-scale servo tasks, the nano-manipulators are required to deliver micro/nano precision movements with additional design specifications such as high speed, large workspace and compact size, which poses major research challenges for both mechanism designs and control algorithms [3,4].

The design and control of nano-manipulating systems have attracted more and more research efforts in recent years. From design perspectives, most of the XYZ nano-manipulators are constructed by stacking three one-degree-of-freedom (1-DOF) nano-stages [5]. Although the serial structure enables a relatively simple control implementation, it usually leads to an unbalanced and bulky structure, which may limit the accuracy and speed of the manipulating systems. Alternatively parallel-kinematic architecture-based multi-axial nano-manipulators have been investigated, such as [2], where high

\footnotetext{
* Corresponding author at: Key Laboratory of High-efficiency and Clean Mechanical Manufacturing, Ministry of Education, School of Mechanical Engineering, Shandong University, Jinan 250061, China.

E-mail address: pengyan2007@gmail.com (P. Yan).
} 
bandwidth (larger than $1 \mathrm{kHz}$ ) and small workspace (less than $20 \mu \mathrm{m}$ ) are obtained. To achieve larger travel range, various designs, such as multiple piezo actuators in series connection per DOF [6] and mechanical amplification [7], have been developed effectively, with inevitable compromise of size, power consumption, and drop of mechanical resonance frequencies.

Regardless of the mechanical designs to enlarge the actuation stroke, the nano-manipulators are still likely to trigger actuator saturations when performing large range trajectory tracking. The existence of disturbances (which is almost inevitable in nano-scale control systems) and system uncertainties will further deteriorate the situation of saturation, which has significant adverse effects on control system performance and possible mechanical failures [8]. Various control approaches have been developed to deal with actuator saturations, such as the parametric discrete-time periodic Lyapunov equation based method [9], the decoupled anti-windup structure [10,11], the internal model based anti-windup compensator [12] and the anticipatory anti-windup scheme [13], to name just a few. However most of the existing results on saturation control are discussed for general control systems without considering the specific properties of tracking control structures, such as internal model based control architecture [14], repetitive control [15] and adaptive backstepping control [16]. With this motivation, our recent work [17] proposed a novel robust anti-windup tracking control architecture by combining a parallel internal-model structure with a robust anti-windup compensator to handle the saturation nonlinearity and the unmodeled dynamics, such that high precision tracking can be achieved.

In the present paper, the design and tracking control problem of an XYZ nano-manipulator is studied to support the need of surface plasmon (SP) confocal microscope, where the target sample is placed on the nano-manipulator to precisely follow given trajectories during operation. By means of theoretical analysis and FEA (finite-element analysis), an XYZ nanomanipulator with large workspace and high mechanical bandwidth is designed to support large stroke high precision trajectory tracking. Meanwhile, a robust anti-windup tracking control architecture is deployed on the designed nano-manipulator to achieve high precision tracking in the presence of saturations. As a theoretical extension on our previous result [17], the robust anti-windup compensator design is formulated as a two block $\mathcal{H}_{\infty}$ optimization problem and further solved by the Nehari approach. Detailed controller design procedure is illustrated by a trajectory tracking example.

The remainder of the paper is organized as follows. In Section 2, the design and mathematical modeling of the nanomanipulator is described. The control strategy with a robust anti-windup tracking controller is discussed in Section 3, where an improved $\mathcal{H}_{\infty}$ optimization algorithm for the robust anti-windup compensator design is given. As a specific case of tracking sinusoidal references, the controller design is detailed in Section 4. Experimental results for controlling the designed nano-manipulator are demonstrated in Section 5, followed by some concluding remarks in Section 6.

\section{Mechanical design and modeling}

\subsection{Design motivation}

The motivation of this work is to support a ultramicroscopic imaging research project by the SP confocal microscope as depicted in Fig. 1, where the sample is placed on the XYZ nano-manipulator to move on the focal plane of the objective. In particular, the $\mathrm{XYZ}$ nano-stage adjusts the sample along the $\mathrm{Z}$ direction to the focal plane and then carries the sample to accomplish the $\mathrm{XY}$ planar scanning motions by following given trajectories. Based on the scanning range and speed requirements, the XYZ nano-manipulator is expected to satisfy the following objectives: (1) having a workspace of the nano-stage larger than $70 \mu \mathrm{m}$; (2) with a natural frequency higher than $500 \mathrm{~Hz}$.

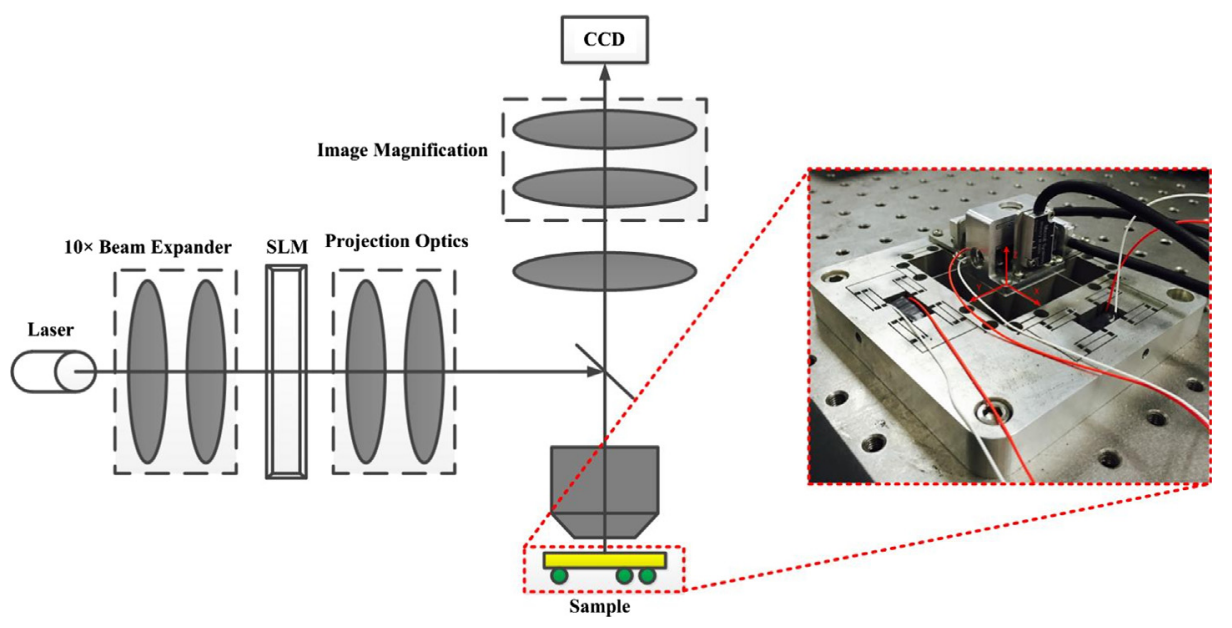

Fig. 1. Simplified schematic diagram of the experimental setup of a SP confocal microscope. 


\subsection{Mechanical design of the nano-stage}

Before presenting the main results on the modeling and tracking control of such system, we would like to start with a brief sketch of the mechanical design. As shown in Fig. 1, the proposed flexure-based XYZ nano-manipulator consists of a parallel XY nano-stage and a $Z$ nano-stage mounted onto the XY platform. Note that the $Z$ nano-stage, as depicted in Fig. 2, is an improvement on our previous design [18], with similar architecture consisting of a bridge-type displacement amplification mechanism and a leaf-springs-based guiding mechanism. Therefore we focus on the design and analysis of the XY nano-stage in this paper.

As illustrated in Fig. 3, the XY nano-stage is composed of two main sections. The outer section consists of two compound bridge-type amplifiers to amplify the output displacements of the piezoelectric actuators. The inner section consists of an XY motion platform based on parallel 4-PP (prismatic) structure. The symmetric parallel 4-PP structure improves the stage stiffness and suppresses the disturbance from manufacturing tolerances, temperature variation and assembly errors. Two type $\mathrm{P}$ joints, as shown in Fig. 4, are adopted in this design. The first type P joint, as illustrated in Fig. 4(a), plays the role of motion decoupling by employing single-notch right circular flexure hinges to improve the static stiffness and avoid the parasitic movement. The second type P joint, as shown in Fig. 4(b), acts as the guiding mechanism, where leaf-spring flexures replace the notch flexure hinges and the rigid links to improve the motion capability of the joint.

\subsection{Static modeling of the XY nano-stage}

Based on the pseudo-rigid-body-model (PRBM) method, the equivalent torsional spring models of the guiding leaf-spring flexures and decoupling four-bar parallelogram are illustrated in Fig. 5. Referring to [19], the equivalent rotational stiffness $k_{r 1}$ of the leaf-spring flexures and $k_{r 2}$ of the single-notched right circular flexure hinges can be expressed as

$$
\begin{aligned}
k_{r 1} & =2 \gamma K_{\Theta} \frac{E b_{1} t_{1}^{3}}{l_{1}} \approx \frac{2 \pi E b_{1} \gamma^{2} t_{1}^{3}}{l_{1}}, \\
k_{r 2} & =\frac{\sqrt{2} E b_{2} t_{2}^{5 / 2}}{9 \pi r^{1 / 2}}
\end{aligned}
$$

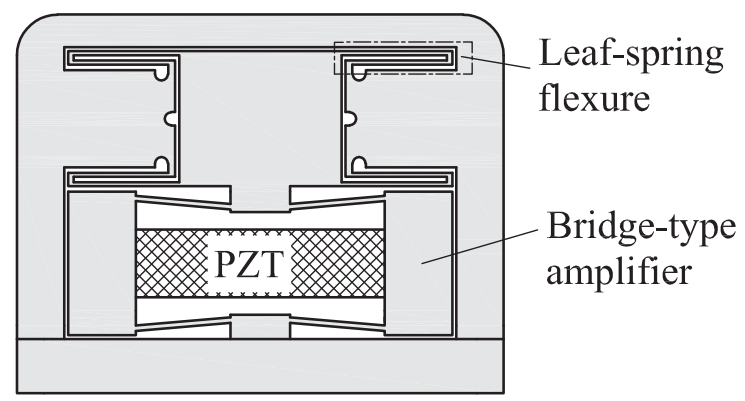

Fig. 2. Schematic diagram of the $\mathrm{Z}$ nano-stage.

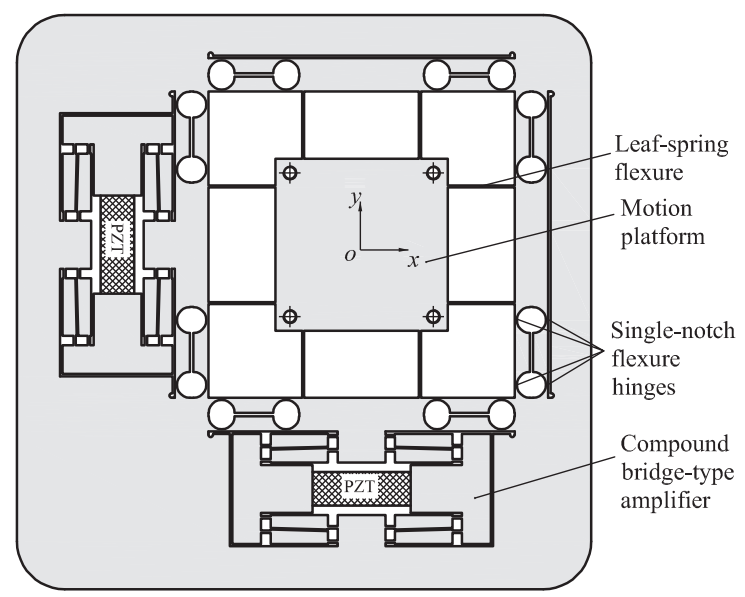

Fig. 3. Schematic diagram of the $\mathrm{X}-\mathrm{Y}$ nano-stage. 


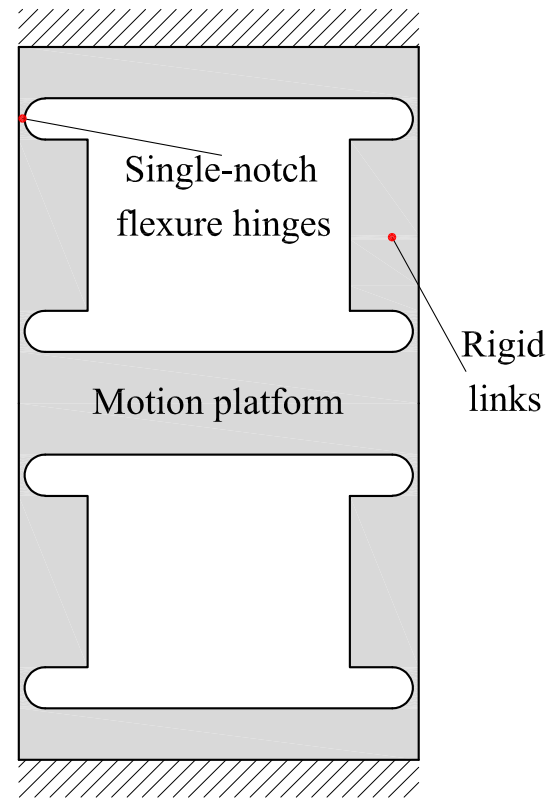

(a)

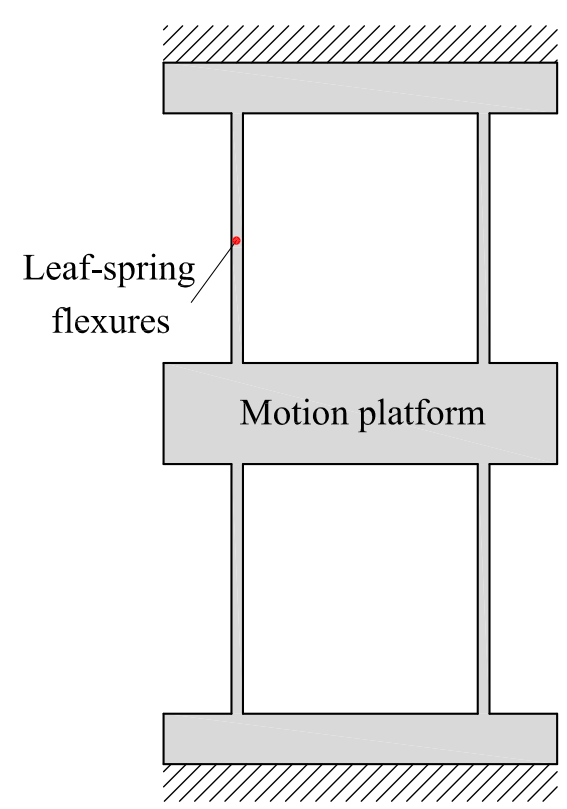

(b)

Fig. 4. Two types of prismatic joints. (a) Based on the notch flexure hinges and rigid links. (b) Based on leaf-spring flexures.
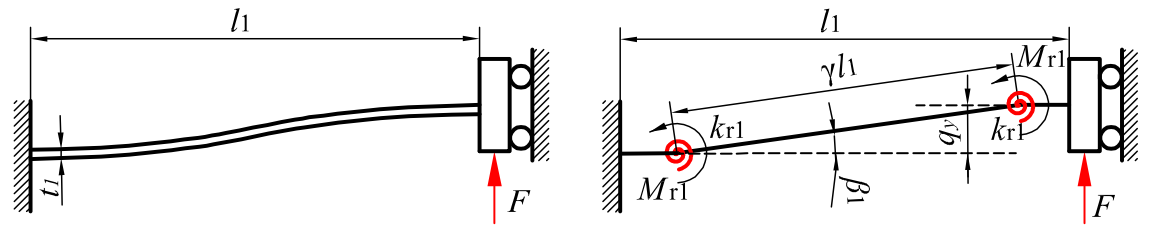

(a)
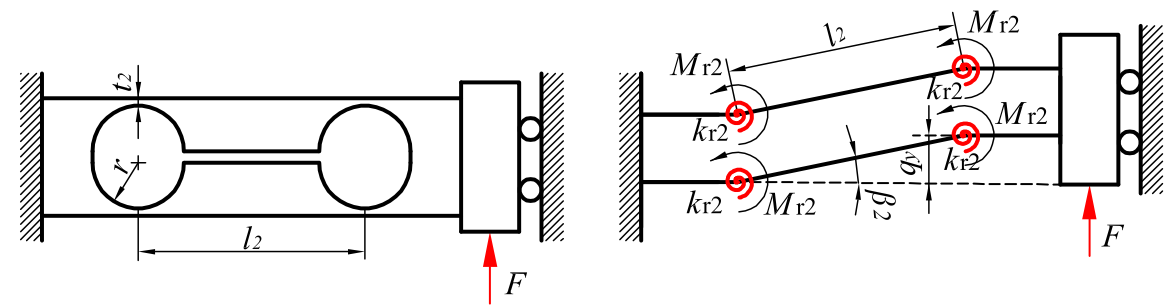

(b)

Fig. 5. Equivalent torsional spring model. (a) Guiding leaf-spring flexures. (b) Decoupling four-bar parallelogram.

where $l_{1}, b_{1}$ and $t_{1}$ are the length, width and thickness of the leaf-spring flexures, $b_{2}, t_{2}$ and $r$ are the width, thickness and radius of the single-notched hinges, $E$ is the material's Young's modulus, $\gamma \approx 0.8517$ is the characteristic radius factor, the product $\gamma l_{1}$ is the length of pseudo-rigid-body links, and $K_{\Theta} \approx \pi \gamma$ is the stiffness coefficient.

Accordingly, the equivalent torsional spring model of the guiding and decoupling mechanisms in one direction is shown in Fig. 6. Based on the geometric relationships and virtual work principle, we have the following relationships:

$$
\begin{aligned}
& q_{y}=l_{2} \tan \beta_{2}=\gamma l_{1} \tan \beta_{1}, \\
& F_{y} q_{y}=16 k_{r 1} \beta_{1}^{2}+8 k_{r 2} \beta_{2}^{2},
\end{aligned}
$$

where $q_{y}$ is the output displacement of the motion platform, $F_{y}$ is the driving force, $l_{2}$ is the length of the rigid link between two single-notched right circular flexure hinges, $\beta_{1}$ and $\beta_{2}$ are the rotation angel of the leaf-spring flexures and single-notched right circular flexure hinges respectively. 


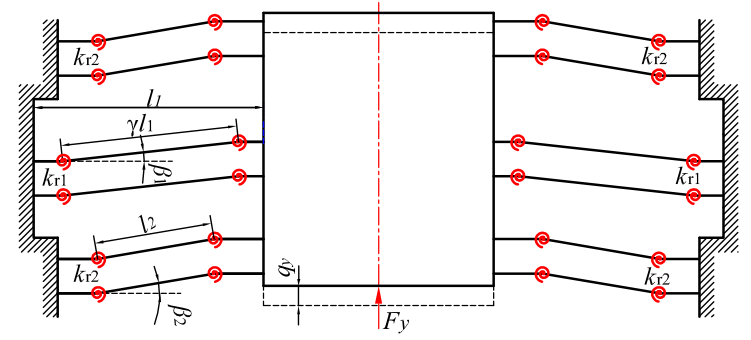

Fig. 6. Equivalent torsional spring model of the guiding and decoupling mechanisms.

Then, we can calculate the equivalent stiffness $k_{m}$ of the guiding and decoupling mechanism as

$$
k_{m}=\frac{F_{y}}{q_{y}}=\frac{8 k_{r 1}}{\gamma^{2} l_{1}^{2}}+\frac{16 k_{r 2}}{l_{2}^{2}} .
$$

For the compound bridge-type amplifier, the corner-filleted flexure hinges are adopted because they are more flexible than other type flexure hinges of the same size. We assume that each flexure hinge has two types of stiffness: translational $k_{l}$ and rotational $k_{r}$, which are approximated as

$$
\begin{aligned}
& k_{l} \approx \frac{E b_{3} t_{3}}{l_{3}}, \\
& k_{r} \approx \frac{E b_{3} t_{3}^{3}}{12 l_{3}},
\end{aligned}
$$

where $l_{3}, b_{3}$ and $t_{3}$ are the length, width, and thickness of the corner-filleted flexure hinges.

Accordingly, the simplified spring model of the amplifier is depicted in Fig. 7(a). Because of the symmetrical structure, only one bridge arm, as shown in Fig. 7(b), is analyzed to establish the mathematical model. According to the geometric relationships and force equilibrium, we have

$$
\begin{aligned}
& \delta x=\delta l \cdot \cos \alpha+\delta \alpha \cdot l \sin \alpha, \\
& \delta y=\delta \alpha \cdot l \cos \alpha-\delta l \cdot \sin \alpha, \\
& F_{a x}=\frac{1}{4} F_{i n}, \\
& F_{a y}=\frac{1}{4} F_{y}, \\
& F_{l}=F_{a x} \cos \alpha+F_{a y} \sin \alpha, \\
& M_{r}=\frac{F_{a x} l \sin \alpha-F_{a y} l \cos \alpha}{2},
\end{aligned}
$$

where $l$ is the length of the single arm, $\alpha$ is angle between the rigid links and the horizontal line, $\delta \alpha$ and $\delta l$ are the rotation angle and linear deformation of the arm, $\delta x$ and $\delta_{y}$ are the input and output displacements, $F_{a x}, F_{a y}$ and $F_{l}$ are the input force, output load and internal force applied to the arm, and $M_{r}$ is internal moment generated at the rotational pivot of the flexure hinge.

According to the virtual work principle the following relationship is obtained

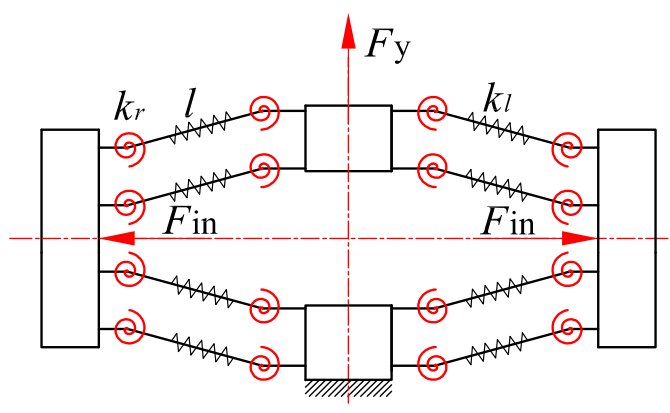

(a)

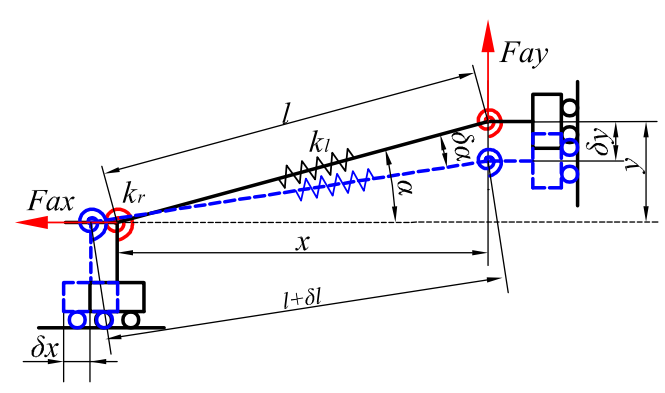

(b)

Fig. 7. Equivalent spring model of the compound bridge-type amplifier. (a) The compound bridge-type amplifier. (b) The single arm of the amplifier. 


$$
F_{a x} \cdot \delta x=2 M_{r} \cdot \delta \alpha+F_{a y} \cdot \delta y+F_{l} \cdot \delta l,
$$

By substituting $F_{y}=k_{m} q_{y}$, we can derive the displacement amplification ratio $R_{\mathrm{amp}}$ and input stiffness $k_{\text {in }}$ of the stage as

$$
\begin{aligned}
& R_{\mathrm{amp}}=\frac{4 k_{r} k_{l}}{A C+B}, \\
& k_{\mathrm{in}}=\frac{4 k_{r} k_{l}-k_{m} R_{\mathrm{amp}}\left(2 k_{r}-l^{2} k_{l}\right) \sin \alpha \cos \alpha}{2 k_{r} \cos ^{2} \alpha+k_{l} l^{2} \sin ^{2} \alpha},
\end{aligned}
$$

where

$$
\begin{aligned}
& A=\frac{2 k_{r} \cos ^{2} \alpha+k_{l} l^{2} \sin ^{2} \alpha}{\left(k_{l} l^{2}-2 k_{r}\right) \sin \alpha \cos \alpha}, \\
& B=k_{m}\left(2 k_{r}-l^{2} k_{l}\right) \sin \alpha \cos \alpha, \\
& C=4 k_{r} k_{l}+k_{m}\left(k_{l} l^{2} \cos ^{2} \alpha+2 k_{r} \sin ^{2} \alpha\right) .
\end{aligned}
$$

In this design, the material of the nano-stage is chosen as aluminum alloy Al7075-T6 due to its high ratio of yield strength. It is noticeable that the performance of the nano-stage is highly dependent on some dimensional parameters. According to the above equations and additional considerations on the constraints of allowable stress, size limitation, and the features of the piezo actuators, the dimensional parameters of the nano-stage are optimized as shown in Table 1. As a result, the proposed nano-stage achieves a displacement amplification ratio of 5.16.

\subsection{Finite element analysis (FEA)}

Furthermore static and modal analysis is carried out by FEA method to verify the design. The static analysis results are illustrated in Fig. 8. It is clear that the developed X-Y nano-stage has an amplification ratio of 4.95 according to the FEA results, which is fairly consistent with the theoretical value. The tiny displacement loss is mainly due to the reason that the links of the compliant mechanisms are not rigid bodies, thus generate deformations under stress. Meanwhile the first four mode shapes extracted by ANSYS are shown in Fig. 9, and the corresponding frequencies are listed in Table 2. The modal analysis results demonstrate that first resonant frequencies of the stage along the $\mathrm{X}$ and $\mathrm{Y}$ axes both are around $520 \mathrm{~Hz}$.

\section{Control strategy}

Ultra high precision trajectory tracking is one of the central topics in nano-manipulating systems attracting significant research efforts in recent years. The control challenge is further complicated by actuator saturation, which is almost inevitable for piezoelectric actuators. Recall that in our recent result [17], a robust anti-windup tracking controller has been developed to achieve tracking performance with the saturation compensation capability, where a robust anti-windup compensator is introduced on top of the parallel internal-model tracking controller to adjust the stabilizer outputs and system

Table 1

Structure parameters of the nano-stage $(\mathrm{mm})$.

\begin{tabular}{lllllllll}
\hline$l$ & $l_{1}$ & $t_{1}$ & $b_{1}$ & $l_{2}$ & $r$ & $b_{3}$ & $b_{3}$ \\
\hline 12 & 14 & 0.4 & 15 & 13.5 & 2.75 & 15 & 2 \\
\hline
\end{tabular}

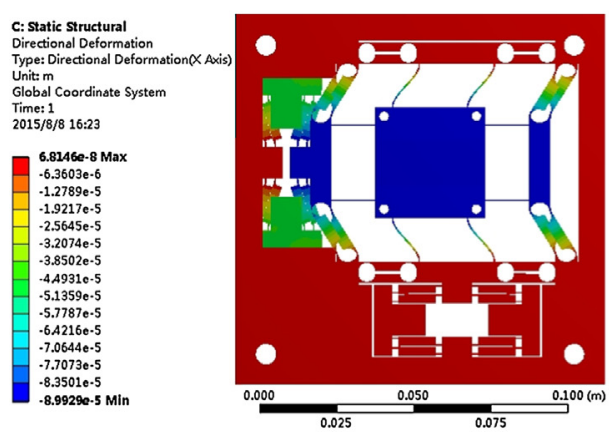

(a)

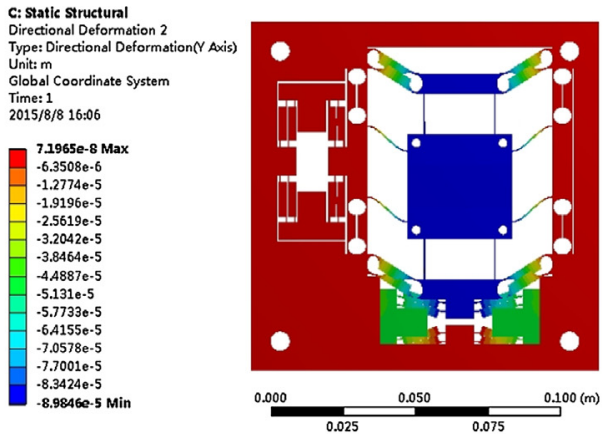

(b)

Fig. 8. Static analysis results of the nano-stage obtained by ANSYS. (a) X-direction. (b) Y-direction. 


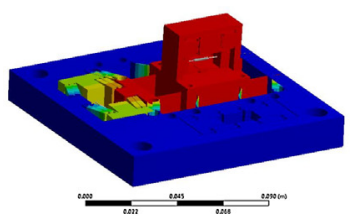

(a)

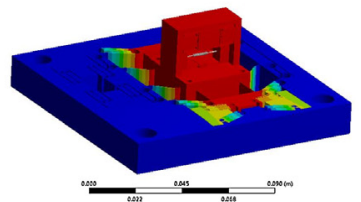

(b)

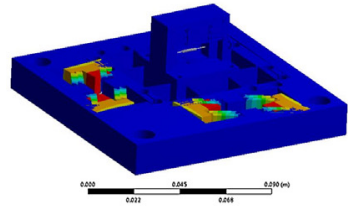

(c)

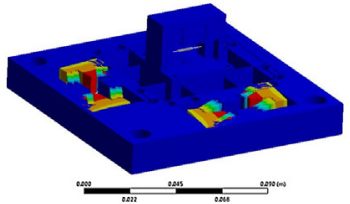

(d)

Fig. 9. First four modes of the nano-stage obtained by ANSYS. (a) 1st mode. (b) 2nd mode. (c) 3rd mode. (d) 4th mode.

Table 2

ANSYS results on frequency modes.

\begin{tabular}{llll}
\hline Modes & 1st & 2nd & 3rd \\
\hline Frequency $(\mathrm{Hz})$ & 520.5 & 522.4 & 943.6 \\
\hline
\end{tabular}

measurements. In this section, we extend the above result by developing an optimization design of the anti-windup compensators in the sense of $\mathcal{H}_{\infty}$. The overall control architecture can be depicted in Fig. 10, where the details of each block can be referred to [17].

\subsection{Parallel internal model control structure}

We first recall the design of the parallel internal model based control structure for high precision tracking problem, without considering saturations. As shown in Fig. 11, the tracking signal $r(t)$ considered in the present paper is assumed to be described by the following exogenous dynamical system:

$$
R(s)=\Lambda(s)^{-1} R_{o}(s)
$$

where $R(s)$ is the Laplace transform of $r(t), \Lambda(s)^{-1}$ represents the dynamics of the exogenous system, and $R_{o}(s)$ is the Laplace transform of $r_{o}(t)$, which is typically taken as an impulse to capture initial conditions of the exogenous system $\Lambda(s)^{-1}$.

Lemma 3.1. Suppose that the controller $K(s)$ asymptotically stabilizes the unforced closed-loop system $G_{A}(s)$, then the controller achieves asymptotic tracking performance if the following condition holds:

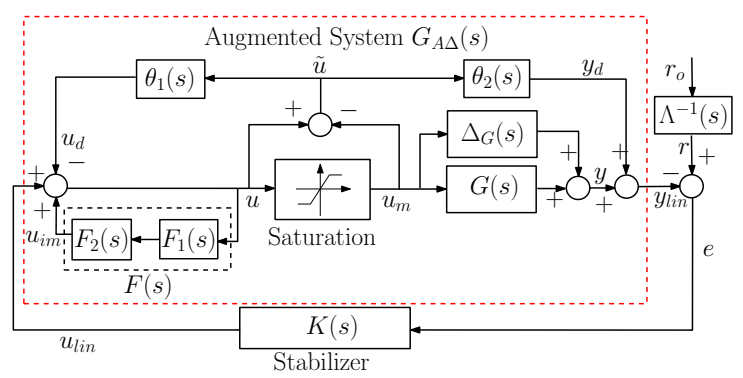

Fig. 10. The anti-windup scheme with the internal model structure.

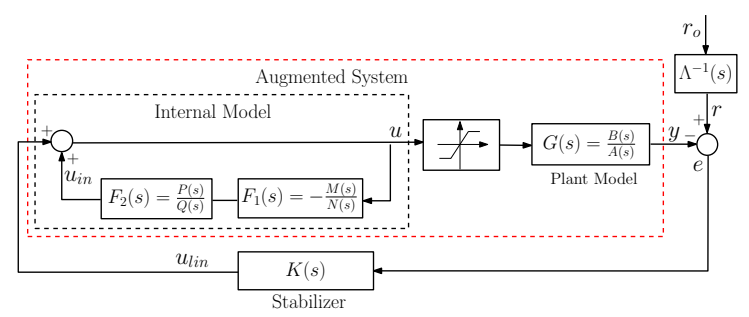

Fig. 11. Block diagram of the parallel internal model control structure. 


$$
\left(1+P(s) Q(s)^{-1} M(s) N(s)^{-1}\right)=A(s)^{-1} \Lambda(s),
$$

where $A(s)$ and $B(s), M(s)$ and $N(s), P(s)$ and $Q(s)$ are defined as the denominator and numerator polynomials of the nominal plant $G(s)$ and internal model units $F_{1}(s)$ and $F_{2}(s)$ respectively, as illustrated in Fig. 11.

Proof. Let

$$
F(s)=F_{1}(s) F_{2}(s)=-\frac{P(s) M(s)}{Q(s) N(s)} .
$$

According to the $\mathrm{I} / \mathrm{O}$ relationship, we have

$$
E(s)=\frac{(1-F(s)) A(s) \Lambda(s)^{-1} R_{o}(s)}{(1-F(s)) A(s)+B(s) K(s)} .
$$

Hence,

$$
\lim _{t \rightarrow \infty} e(t)=\lim _{s \rightarrow 0} s E(s)=\lim _{s \rightarrow 0} \frac{s(1-F(s)) A(s) \Lambda(s)^{-1} R_{o}(s)}{(1-F(s)) A(s)+B(s) K(s)} .
$$

Note that $R_{o}(s)=1$ as $r_{o}(t)$ is an impulse signal. It is straightforward that $(1-F(s)) A(s)=\Lambda(s)$, which is equivalent to (18), is a sufficient condition to guarantee asymptotic tracking.

We further assume that the plant model has uncertainties in additive form described by $\Delta_{G}(s)$, which is bounded by weighting function $W_{2}(s)$ (i.e. $\left|\Delta_{G}(j \omega)\right|<\left|W_{2}(j \omega)\right|, \forall \omega \in \mathbb{R}$ ). Once the internal model units are constructed, we can obtain the nominal and actual augmented system $\left(G_{A}(s)\right.$ and $\left.G_{A \Delta}(s)\right)$ as

$$
\begin{aligned}
& G_{A}(s)=\frac{G(s)}{1-F(s)}, \\
& G_{A \Delta}(s)=\frac{G(s)+\Delta_{G}(s)}{1-F(s)}=G_{A}(s)(1+\Delta(s)),
\end{aligned}
$$

where $\Delta(s)=\Delta_{G}(s) / G(s)$.

Therefore, the robust stabilizer design can be formulated as the following mixed sensitivity $\mathcal{H}_{\infty}$ problem to optimize both performance requirement and robustness against uncertainties,

$$
\inf _{K s t a b . G_{A}}\left\|\left[\begin{array}{c}
W_{r 1}(s)\left(1+G_{A}(s) K(s)\right)^{-1} \\
W_{r 2}(s)\left(G_{A}(s) K(s)\right)\left(1+G_{A}(s) K(s)\right)^{-1}
\end{array}\right]\right\|_{\infty},
$$

where the definition of weighting functions $W_{r 1}(s)$ and $W_{r 2}(s)$ as well as the design details can be referred to [17].

\subsection{Robust anti-windup compensator}

As illustrated in Fig. 10, the anti-windup structure compensates the actuator saturations by adjusting stabilizer output $u_{\text {lin }}$ with $u_{d}$, and adjusting the plant output $y$ with $y_{d}$ in case of saturations. We define the difference between $u$ and $u_{m}$ as the dead zone operator, which can be approximated by the sector bound as:

$$
\begin{aligned}
& \widehat{d z}(u)=\left\{\begin{array}{l}
u-\sigma_{1}, u \leqslant \sigma_{1} \\
\delta u(\delta \rightarrow 0), \sigma_{1}<u<\sigma_{2}, \\
u-\sigma_{2}, u \geqslant \sigma_{2}
\end{array}\right. \\
& 0<\widehat{d z}(u)<k u,(k=1) .
\end{aligned}
$$

where $\sigma_{1}$ and $\sigma_{2}$ are the saturation values.

Similar to [17], we can redraw Fig. 10 as Fig. 12 under the condition of $\theta_{2}(s)\left(1-F(s)+\theta_{1}(s)\right)^{-1}=G(s)(1-F(s))^{-1}$, with which the augmented system remains identical before and after saturations from I/O perspective. Accordingly, the stability of the original system with saturation nonlinearity in Fig. 10 is equivalent to the stability of the system (mapping $\Gamma: u_{\text {lin }} \rightarrow y_{d}$ ) with dead zone operator depicted in Fig. 13. Based on sector bound criterion, we can derive the robust stability condition for the anti-windup compensators $\theta_{1}(s)$ and $\theta_{2}(s)$ design.

Theorem 3.2. Consider the robust anti-windup tracking control architecture depicted in Fig. 12, where the dead-zone operator replaced by $\widehat{d z}(\cdot)$ given in (23). The closed-loop system is robustly stable if 


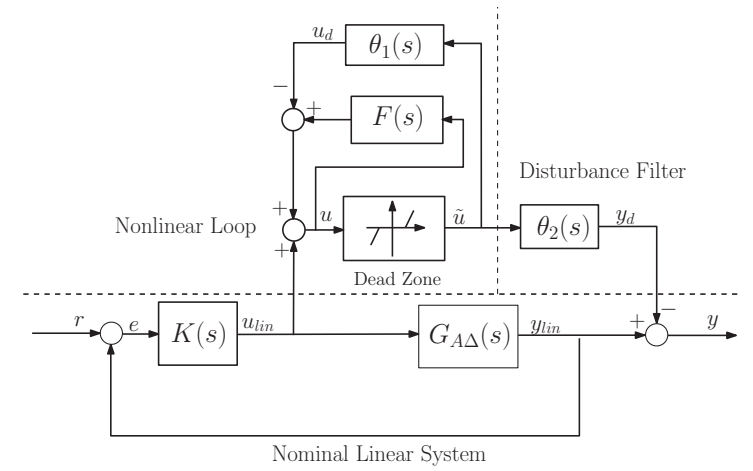

Fig. 12. Equivalent representation of Fig. 10.

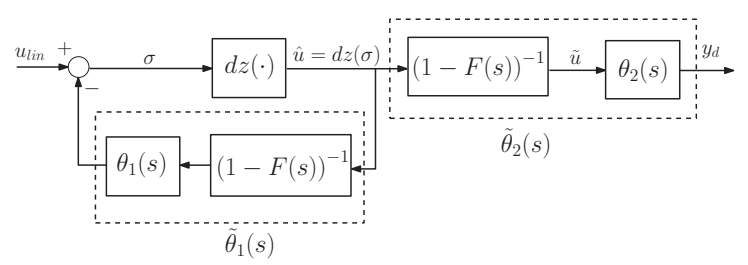

Fig. 13. Equivalent representation of the mapping $\Gamma: u_{\text {lin }} \rightarrow y_{d}$.

$$
\left\|\left[\begin{array}{c}
W_{1}(s) \tilde{\theta}_{1}(s) \\
W_{2}(s)\left(1+\tilde{\theta}_{1}(s)\right)
\end{array}\right]\right\|_{\infty}<1,
$$

where

$$
\begin{aligned}
& \tilde{\theta}_{1}(s)=\theta_{1}(s)(1-F(s))^{-1}, \\
& W_{1}(s)=k(1+\alpha s),(\alpha>0, k=1) .
\end{aligned}
$$

Proof. Recall Popov criterion $[20,21]$ that the feedback structure composed of the operator $\widehat{d z}(\cdot)$ and $\tilde{\theta}_{1}(s)$ illustrated in Fig. 13 is stable if the polar plot of $(1+\alpha s) \tilde{\theta}_{1}(s)+1 / k$ lies to the right of the straight line $\operatorname{Re}=-1 / k$, as shown in Fig. 14 . It is easy to verify that the transfer function $(1-F(s))^{-1}$ is stable. In addition, the stability of filter $\theta_{2}(s)$ can be guaranteed with the fact that $\theta_{1}(s)$ and $(1-F(s))^{-1}$ are stable. Obviously we can obtain a sufficient condition on the stability of the mapping $\Gamma: u_{\text {lin }} \rightarrow y_{d}$ shown in Fig. 13 as

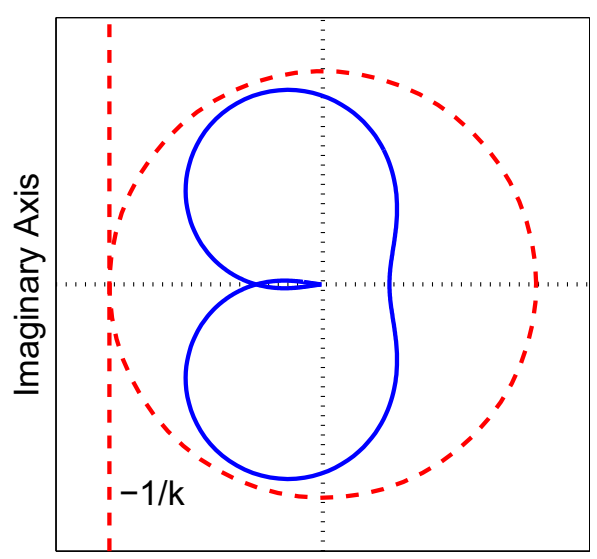

Real Axis

Fig. 14. The Nyquist plot of the Popov criterion. 


$$
\left\|k(1+\alpha s) \tilde{\theta}_{1}(s)\right\|_{\infty}<1
$$

In the meanwhile, considering the existence of system uncertainties, we should rewrite the relationship $\theta_{2}(s)\left(1-F(s)+\theta_{1}(s)\right)^{-1}=G(s)(1-F(s))^{-1}$ as

$$
\frac{\theta_{2 \Delta}(s)}{1-F(s)+\theta_{1}(s)}=\frac{G(s)+\Delta_{G}(s)}{1-F(s)},
$$

It is straightforward that $\theta_{2}(s)$ designed for the nominal system without uncertainties has an error of $\Delta_{\theta_{2}}(s)$ as

$$
\Delta_{\theta_{2}}(s)=\left|\theta_{2}(s)-\theta_{2 \Delta}(s)\right|=\left|\Delta_{G}(s)\left(1+\tilde{\theta}_{1}(s)\right)\right| .
$$

In order to eliminate the adverse effects caused by plant uncertainties and achieve robust stability and tracking performance, we would like to further minimize $\Delta_{\theta_{2}}(s)$ on admissible sets of $\left(\theta_{1}, \theta_{2}\right)$ satisfying relationship (26). Hence we would like to have

$$
\left\|W_{2}(s)\left(1+\tilde{\theta}_{1}(s)\right)\right\|_{\infty}<1
$$

Clearly, Eq. (25) is a sufficient condition of the robust stability condition (26) and (30) of the closed-loop system, which completes the proof.

According to Theorem 3.2, our design objective is to perform the following $\mathcal{H}_{\infty}$ optimization:

$$
\gamma_{\mathrm{opt}}=\inf _{\tilde{\theta}_{1}(s) \in \mathcal{H}_{\infty}}\left\|\left[\begin{array}{c}
W_{1}(s) \tilde{\theta}_{1}(s) \\
W_{2}(s)\left(1+\tilde{\theta}_{1}(s)\right)
\end{array}\right]\right\|_{\infty},
$$

where $\gamma_{\text {opt }}$ is defined as the optimal index.

For the optimization problem (31), we perform spectrum factorization by letting $W_{1}(s) W_{1}(-s)+W_{2}(s) W_{2}(-s)=H(s) H(-s)$, where $H, H^{-1} \in \mathcal{H}_{\infty}$. Then we can define

$$
\begin{aligned}
& R_{1}(s):=W_{2}(s) W_{2}(-s) H(s)^{-1}, \\
& R_{2}(s):=W_{1}(s) W_{2}(s) H(s)^{-1}, \\
& Q_{1}(s):=H(s) \tilde{\theta}_{1}(s) .
\end{aligned}
$$

Moreover, we construct

$$
\begin{aligned}
& \gamma^{2}-R_{2}(-s) R_{2}(s)=R_{\gamma}(-s) R_{\gamma}(s), \\
& R_{\gamma}^{-1}(s) R_{1}(s)=R_{+}(s)+R_{-}(s), \\
& Q(s)=-R_{\gamma}^{-1}(s) Q_{1}(s)-R_{-}(s),
\end{aligned}
$$

where $\gamma$ is the index to be minimized satisfying

$$
\inf _{\tilde{\theta}_{1}(s) \in \mathcal{H}_{\infty}}\left\|\left[\begin{array}{c}
W_{1}(s) \tilde{\theta}_{1}(s) \\
W_{2}(s)\left(1+\tilde{\theta}_{1}(s)\right)
\end{array}\right]\right\|_{\infty} \leqslant \gamma,
$$

and $R_{\gamma}, R_{\gamma}^{-1} \in \mathcal{H}_{\infty}, R_{-}(s) \in \mathcal{H}_{\infty}$ and $R_{+}(s)$ has all poles in $\mathbb{C}_{+}$(the open right half of the complex plane).

With the above notations, the two block optimization problem (31) can be turned into the following one block Nehari problem

$$
\gamma_{\mathrm{opt}}=\inf _{\tilde{\theta}_{1}(s) \in \mathcal{H}_{\infty}}\left\|R_{+}(s)-Q(s)\right\|_{\infty}
$$

Considering that $R_{+}(s)$ is finite-dimensional in this paper, we assume that $R_{+}(s)=C_{+}\left(s I+A_{+}\right)^{-1} B_{+}$. Thus all the eigenvalues of the matrix $A_{+}$are in $\mathbb{C}_{-}$(the open left half of the complex plane). Therefore, we can construct the Lyapunov equations as

$$
\left\{\begin{array}{l}
A_{+} P_{+}+P_{+} A_{+}^{T}+B_{+} B_{+}^{T}=0 \\
A_{+}^{T} Q_{+}+Q_{+} A_{+}+C_{+}^{T} C_{+}=0
\end{array}\right.
$$

where $P_{+}$and $Q_{+}$are the solutions of the Lyapunov equations. Accordingly, the solution to the Nehari problem (38) can be derived by the following lemma.

Lemma 3.3. (Francis [22]) Let $\lambda\left(P_{+} Q_{+}\right)$denote the eigenvalue of the matrix $P_{+} Q_{+} \cdot \gamma_{\text {opt }}$ is the smallest $\gamma>0$ such that $\lambda_{\max }\left(P_{+} Q_{+}\right) \leqslant 1$. Furthermore, the optimal index $\gamma_{\mathrm{opt}}$ and compensator are determined by 


$$
\begin{aligned}
& \theta_{1}(s)=-H(s)^{-1} R_{r}(s)\left(Q_{\mathrm{opt}}(s)+R_{-}(s)\right)(1-F(s)), \\
& \theta_{2}(s)=G(s)\left(1+\theta_{1}(s)(1-F(s))^{-1}\right),
\end{aligned}
$$

where

$$
Q_{\mathrm{opt}}(s)=R_{+}(s)-\frac{\gamma_{\mathrm{opt}}^{2} C_{+}\left(s I+A_{+}\right)^{-1} x_{\max }}{B_{+}^{T}\left(s I-A_{+}^{T}\right)^{-1} Q_{+} x_{\max }} .
$$

\section{Modeling and controller design}

\subsection{Prototype stage and experimental apparatus setup}

Based on the design and analysis results presented in Section 2, a prototype of the propsed nano-manipulator is monolithically machined and an experimental setup is established, as depicted in Fig. 15. Piezo stack actuators (from Noliac Group) with a free stroke of $27 \mu \mathrm{m}$ are used to drive the nano-stage. Considering the high bandwidth and high precision requirements of the piezoelectric actuators, high bandwidth voltage amplifiers are designed to drive the piezo stacks. Accordingly linear encoders (from MicroE Systems) with a resolution of $1.2 \mathrm{~nm}$ are instrumented as the displacement sensors to generate real time position signals. Feedback control implementations are deployed using a dSPACE R1103 rapid prototyping system. A sampling frequency of $20 \mathrm{kHz}$ is chosen to avoid possible aliasing effects during the experiments and ensure high bandwidth control implementations.

\subsection{Modeling and system parameters identification}

As the nano-stage is actuated to track a certain trajectory along the XY plane, the device is interpreted as a linear system with two inputs and two outputs

$$
Y_{d}(s)=G(s) U(s)=\left[\begin{array}{ll}
G_{x x} & G_{x y} \\
G_{y x} & G_{y y}
\end{array}\right] U(s),
$$

where $Y_{d}(s)$ is the Laplace transform of the output displacements along the $\mathrm{X}$ and $\mathrm{Y}$ axes $\left[d_{x}, d_{y}\right]^{T}, U(s)$ is the Laplace transform of the applied voltage signals $\left[u_{x}, u_{y}\right]^{T}$, and $G(s)$ is the transfer function from the inputs $\left[u_{x}, u_{y}\right]^{T}$ to the outputs $\left[d_{x}, d_{y}\right]^{T}$. The blue solid lines in Fig. 16 show the magnitude and phase responses of the individual transfer functions of $G(s)$. Data for these plots were obtained by applying swept sine signals along $\mathrm{X}$ and $\mathrm{Y}$ axes and recording the corresponding encoder responses $d_{x}$ and $d_{y}$. It is apparent from the red dashed line of Fig. 16 that $G_{x x}(s)$ and $G_{y y}(s)$ can be nicely modeled as third-order systems:

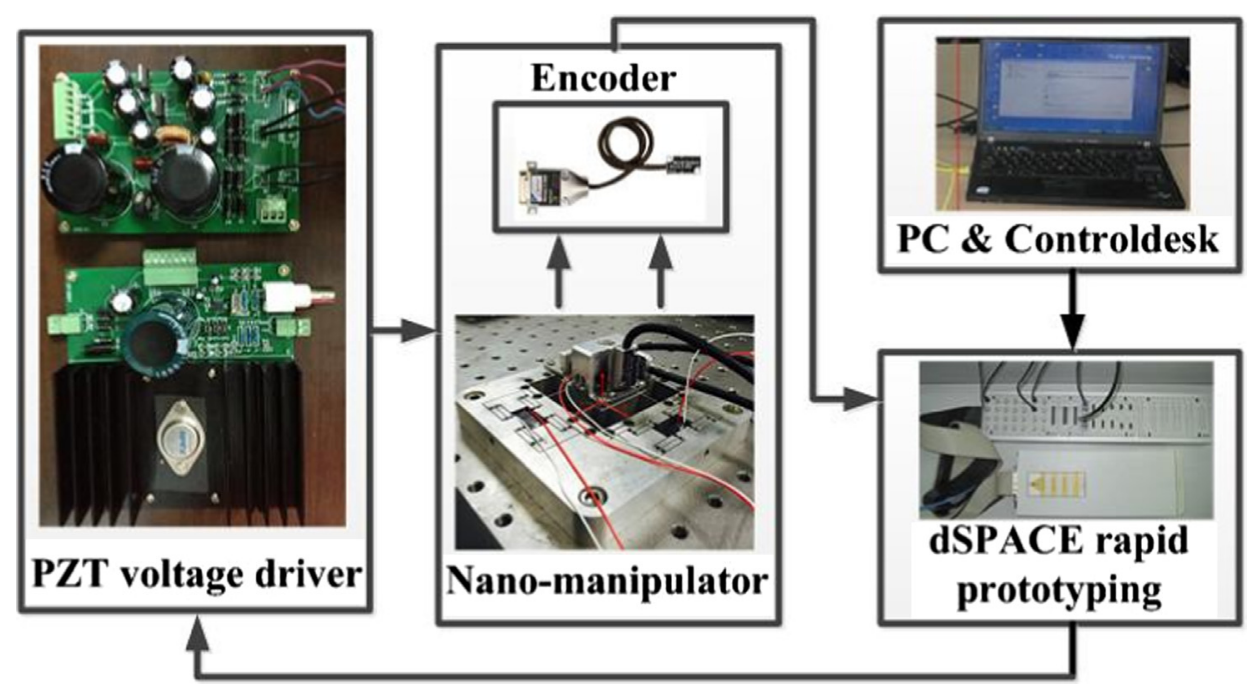

Fig. 15. Experimental setup of nano-manipulating system. 

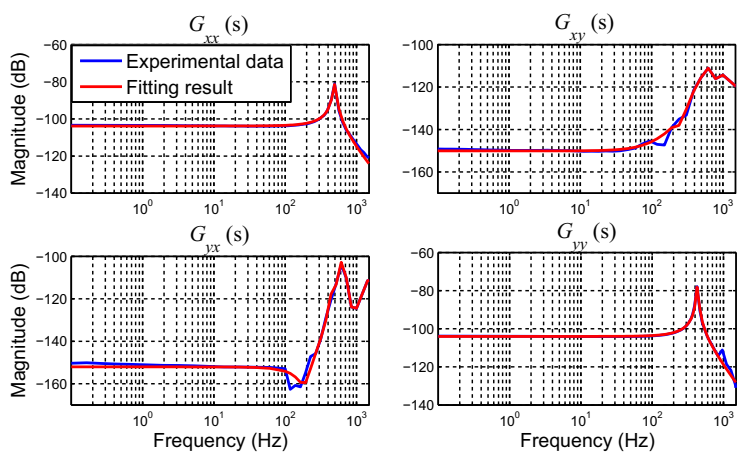

(a)
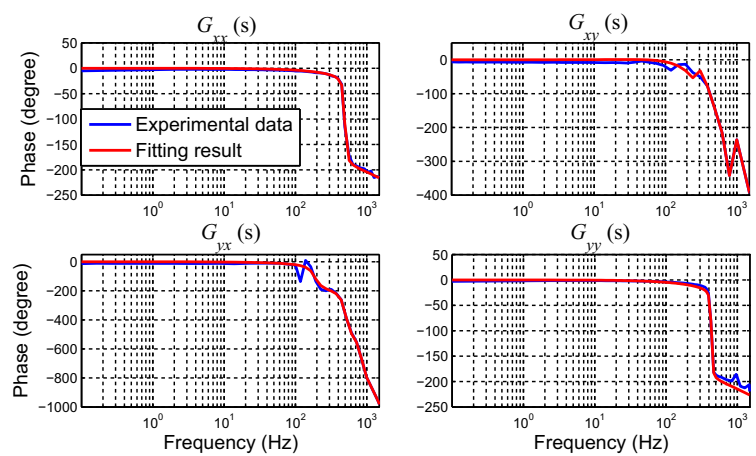

(b)

Fig. 16. Open-loop frequency responses. (a) Magnitude responses. (b) Phase responses.

$$
\begin{aligned}
G_{x x}(s) & =\frac{76200}{s^{3}+12600 s^{2}+1.24 \times 10^{7} s+1.18 \times 10^{11}}, \\
G_{y y}(s) & =\frac{40400}{s^{3}+8781 s^{2}+8.01 \times 10^{7} s+6.45 \times 10^{11}} .
\end{aligned}
$$

It is also very clear that the cross-coupling effects of $G_{x y}(s)$ and $G_{y x}(s)$ are negligible with respect to $G_{x x}(s)$ and $G_{y y}(s)$, as shown in Fig. 16. Thus the motions on $\mathrm{X}$ and $\mathrm{Y}$ directions can be controlled separately in a decoupled fashion.

\subsection{Controller design}

We would like to sketch the design of the proposed control architecture by studying a $50 \mathrm{~Hz}$ tracking example of the nano-stage since it is more challenging to track high-frequency signals. Assume that the desired reference trajectory is $r(t)=35 \sin (100 \pi t+\pi / 2)+35(\mu \mathrm{m})$. We have the exogenous dynamics as:

$$
\Lambda(s)=s^{2}+10000 \pi^{2} .
$$

In what follows we would like to investigate the design of the internal model units. Note that there are multiple ways to construct the internal model units satisfying (18). A straightforward one is to set $M(s)=B(s), N(s)=A(s), Q(s)=1, P(s)=\frac{\Lambda(s)-A(s)}{B(s)}$.

According to the identified plant models (44) and (45), the internal model units can be calculated as

$$
\begin{aligned}
& F_{1 x}(s)=-\frac{76200}{s^{3}+12600 s^{2}+1.24 \times 10^{7} s+1.18 \times 10^{11}}, \\
& F_{2 x}(s)=-\left(\frac{\Lambda(s)}{76200}-\frac{1}{F_{1}(s)}\right) \\
& F_{1 y}(s)=-\frac{40400}{s^{3}+8781 s^{2}+8.01 \times 10^{7} s+6.45 \times 10^{11}}, \\
& F_{2 y}(s)=-\left(\frac{\Lambda(s)}{40400}-\frac{1}{F_{1}(s)}\right) .
\end{aligned}
$$

The robust stabilizer can be synthesized for the augmented system $G_{A}(s)$ based on the requirements on robustness and performance. Based on the mixed sensitivity optimization approach for $\mathcal{H}_{\infty}$ controller design, we can derive the 6 order robust stabilizers as

$$
\begin{aligned}
K_{x}(s) & =\frac{3.95 \times 10^{7}\left(s+8.59 \times 10^{4}\right)\left(s+1.15 \times 10^{4}\right)(s+3815.05)\left(s^{2}+1.48 \times 10^{-10} s+9.87 \times 10^{4}\right)}{\left(s+1.24 \times 10^{5}\right)\left(s+7.90 \times 10^{4}\right)\left(s+4.21 \times 10^{4}\right)(s+8982.94)(s+66.50)(s+58.94)}, \\
K_{y}(s) & =\frac{9.64 \times 10^{6}\left(s+8.23 \times 10^{4}\right)\left(s+1.20 \times 10^{4}\right)(s+3491.17)\left(s^{2}+6.48 \times 10^{-11} s+9.87 \times 10^{4}\right)}{\left(s+8.07 \times 10^{4}\right)\left(s+1.12 \times 10^{4}\right)(s+127.05)(s+124.26)\left(s^{2}+4.38 \times 10^{4} s+6.27 \times 10^{8}\right)} .
\end{aligned}
$$

Recall Theorem 3.2 and Lemma 3.3. We need to determine the weighting functions $W_{1}(s)$ and $W_{2}(s)$ to design the robust anti-windup compensator. Particularly, the weighting function $W_{2}(s)$ can be experimentally determined by observing the discrepancy between the experimental measured dynamics and the nominal model. In this design, $W_{1}(s)$ and $W_{2}(s)$ are selected in the following form: 


$$
\begin{aligned}
& W_{1 x}=1+\frac{s}{200 \pi}, \\
& W_{2 x}=\frac{24.85 s+478700}{s^{3}+7094 s^{2}+1.13 \times 10^{7} s+6.40 \times 10^{10}}, \\
& W_{1 y}=1+\frac{s}{200 \pi}, \\
& W_{2 y}=\frac{206.30 s+984900}{s^{3}+2924 s^{2}+7.62 \times 10^{7} s+2.08 \times 10^{10}} .
\end{aligned}
$$

We further recall Eqs. (40) and (41), and derive the reduced order anti-windup compensator $\theta_{1}(s)$ and $\theta_{2}(s)$ as

$$
\begin{aligned}
\theta_{1 x}(s) & =\frac{-1.91 \times 10^{-6}(s-616.93)(s+216.51)\left(s^{2}+9.87 \times 10^{4}\right)\left(s^{2}+131.52 s+8.10 \times 10^{6}\right)}{\left(s+1.24 \times 10^{4}\right)(s+218.11)\left(s^{2}+1252.03 s+3.92 \times 10^{5}\right)\left(s^{2}+0.015 s+8.11 \times 10^{6}\right)\left(s^{2}+226.42 s+9.57 \times 10^{6}\right)}, \\
\theta_{2 x}(s) & =\frac{76200(s+218.12)\left(s^{2}+1252.05 s+3.94 \times 10^{5}\right)\left(s^{2}+0.015 s+8.12 \times 10^{6}\right)}{\left(s+1.24 \times 10^{4}\right)(s+218.11)\left(s^{2}+1252.03 s+3.92 \times 10^{5}\right)\left(s^{2}+0.015 s+8.11 \times 10^{6}\right)\left(s^{2}+226.42 s+9.57 \times 10^{6}\right)}, \\
\theta_{1 y}(s) & =\frac{-1.91 \times 10^{-6}(s-883.37)(s+217.92)\left(s^{2}+2.7 \times 10^{-13} s+9.87 \times 10^{4}\right)\left(s^{2}+2712.31 s+7.59 \times 10^{7}\right)}{(s+8717.47)(s+6600.63)\left(s^{2}+1493.24 s+1.09 \times 10^{6}\right)\left(s^{2}+63.53 s+7.46 \times 10^{6}\right)\left(s^{2}+105.12 s+9.80 \times 10^{6}\right)}, \\
\theta_{2 y}(s) & =\frac{40400(s+6601.01)\left(s^{2}+1493.30 s+1.10 \times 10^{6}\right)\left(s^{2}+105.23 s+9.81 \times 10^{6}\right)}{(s+8717.47)(s+6600.63)\left(s^{2}+1493.24 s+1.09 \times 10^{6}\right)\left(s^{2}+63.53 s+7.46 \times 10^{6}\right)\left(s^{2}+105.12 s+9.80 \times 10^{6}\right)} .
\end{aligned}
$$

Now that we have all the necessary components of the controller structure: the internal model components $F_{1}(s), F_{2}(s)$, the robust anti-windup components $\theta_{1}(s), \theta_{2}(s)$, and the robust stabilizer $K(s)$ respectively. With this, the overall controller can be derived by employing the anti-windup tracking control structure in Fig. 10.

\section{Implementations and experimental results}

Based on the proposed control architecture, hardware-in-loop implementations are comprehensively conducted to evaluate the tracking performance in various scenarios with the sampling frequency of $20 \mathrm{kHz}$. Note that the machined nanomanipulator is mounted on a air-floating platform in order to reduce the external effects including vibrations on the experimental results. For the purpose of improving the reliability of experimental results, each experiment is carried out fifty times. Statistical comparisons are also conducted to validate the proposed control strategy.

\subsection{Tracking performance}

We start with the case of tracking a sinusoidal reference without saturations. As clearly depicted in Fig. 17, the uniaxial tracking error of $e_{\mathrm{rms}}=104 \mathrm{~nm}$ is achieved, where $e_{\mathrm{rms}}$ represents the RMS (Root Mean Square) value of the error signal. The results demonstrate that the proposed robust anti-windup tracking algorithm can achieve the tracking precision with average error less than $0.25 \%$ at the frequency of $50 \mathrm{~Hz}$. As shown in Fig. 18, biaxial contouring is also tested, where the proposed algorithm achieves good performance with less than $0.28 \%$ of the tracking error. The statistical comparisons of both uniaxial

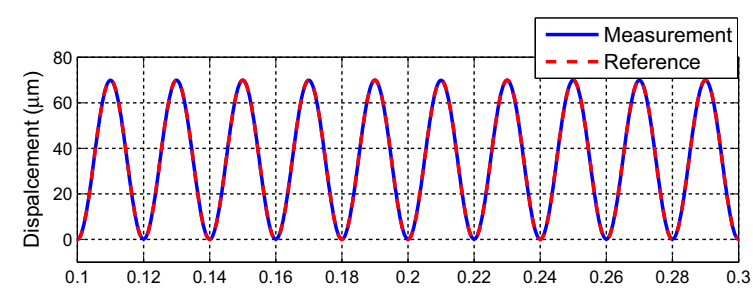

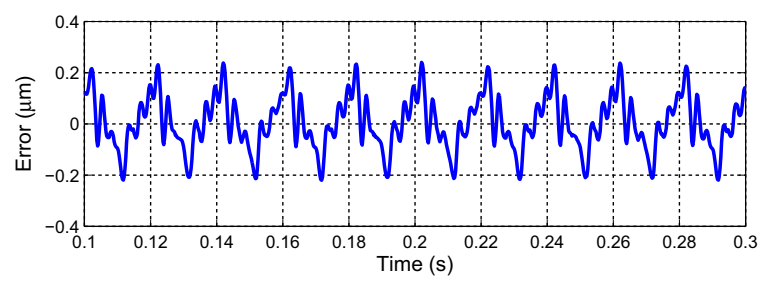

(a)

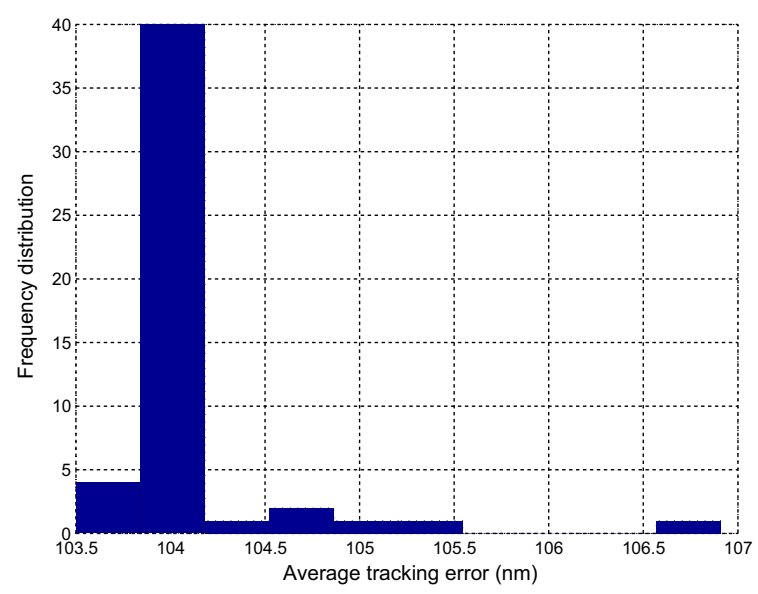

(b)

Fig. 17. Experimental results of tracking a sinusoidal trajectory without saturation. (a) Tracking performance. (b) Statistical comparison of tracking error. 


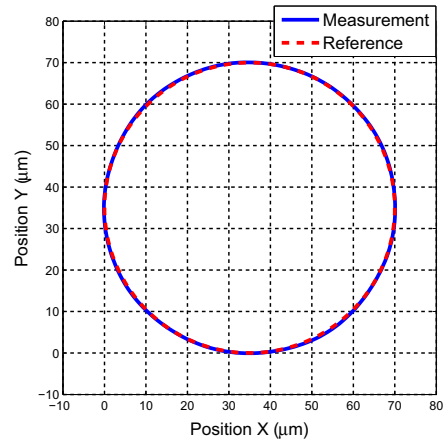

(a)

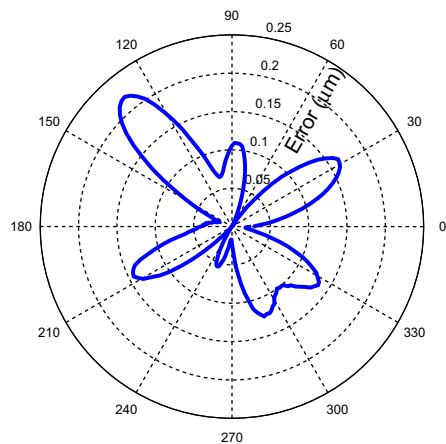

(b)

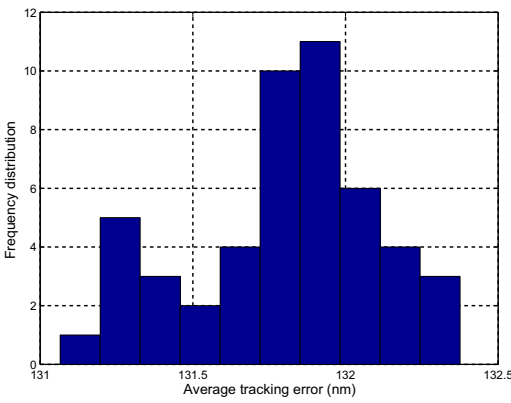

(c)

Fig. 18. Experimental results of biaxial contour tracking without saturation. (a) Tracking a $70 \mu \mathrm{m}$ diameter circle. (b) Tracking errors in polar coordinate. (b) Statistical comparison of tracking error.

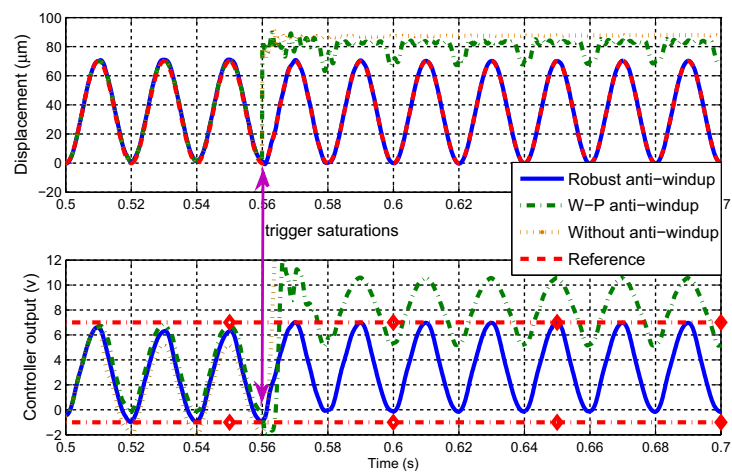

Fig. 19. Experimental results of tracking a sinusoidal trajectory with saturation.

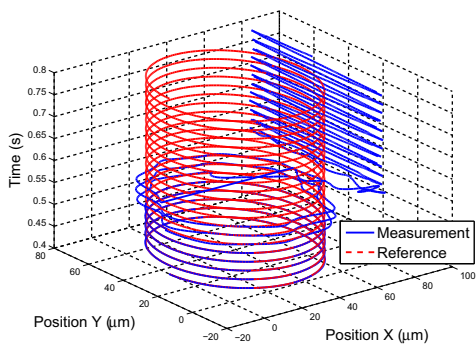

(a)

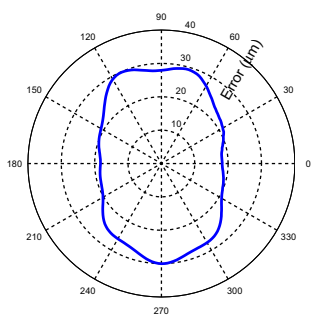

(d)

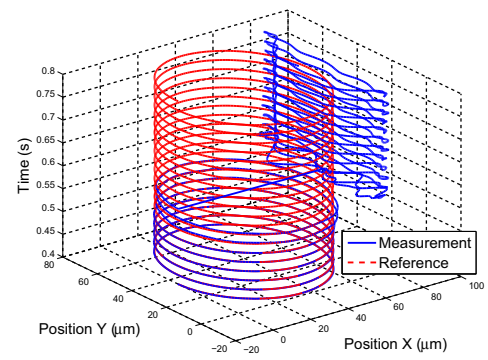

(b)

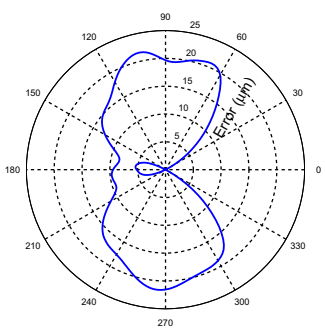

(e)

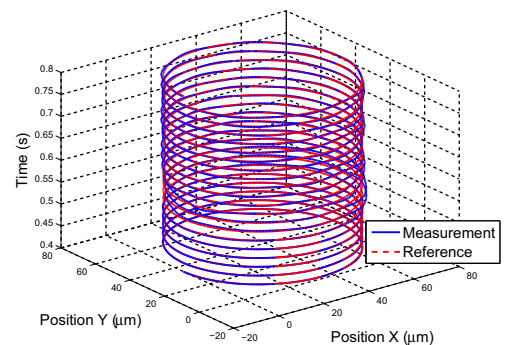

(c)

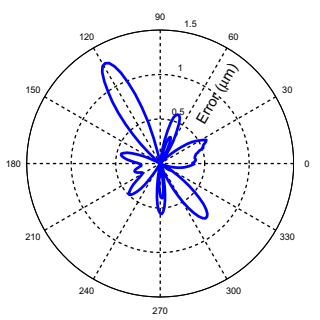

(f)

Fig. 20. Experimental results of biaxial contour tracking. (a) Without anti-windup compensator. (b) W-P anti-windup in [10]. (c) Robust anti-windup. (e) Tracking error with W-P compensator. (f) Tracking error with robust anti-windup compensator. 


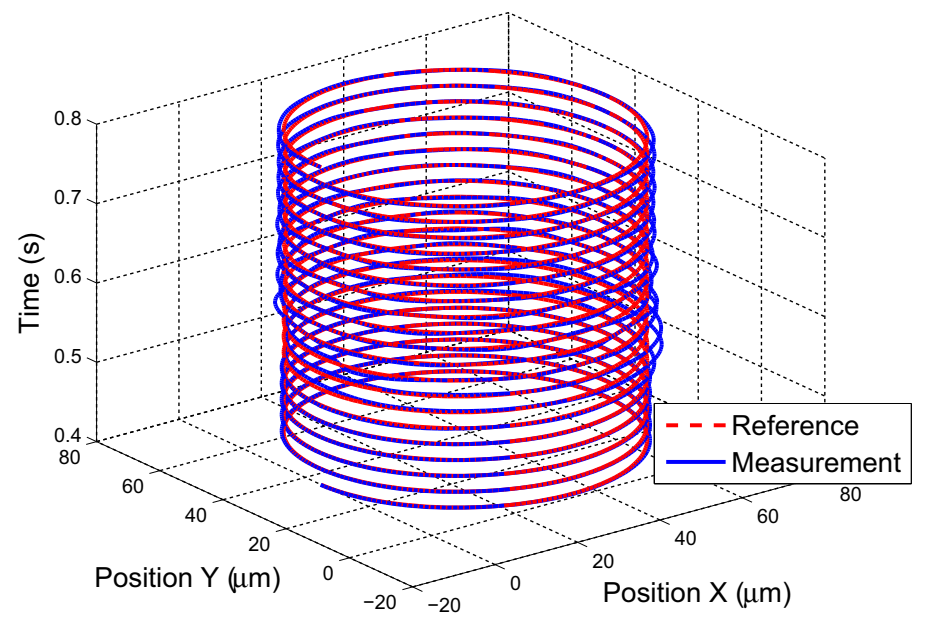

(a)

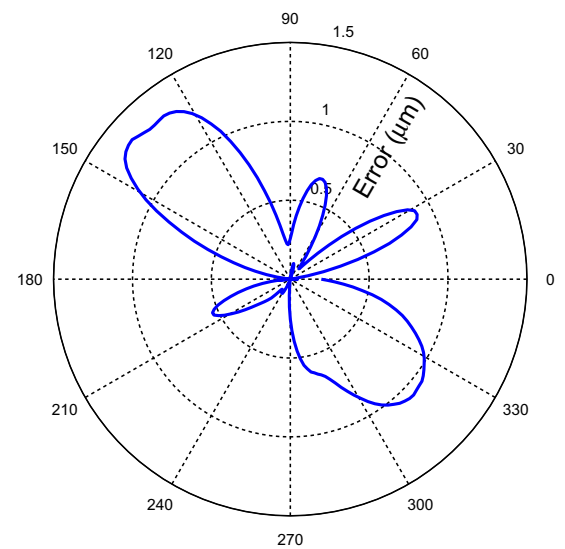

(b)

Fig. 21. Experimental results of tracking performance in the case of carrying a load.

and biaxial tracking errors illustrated in Fig. 17(b) and 18(c) demonstrate good consistency, which validates the effectiveness of the proposed control strategy.

\subsection{Anti-windup performance}

We further add a pulse interference on top of the x-axis control signal to occasionally trigger PZT actuator saturations during the circlar tracking. The uniaxial and biaxial tracking results are illustrated in Figs. 19 and 20. It is straightforward that the internal-model based tracking controller without anti-windup compensator cannot stabilize the system when input saturation occurs, where huge oscillations of control signal and system output are observed. However, with the action of the robust anti-windup compensator, the commanded input converges and the system output achieves asymptotic tracking.

It is also interesting to compare the proposed robust anti-windup compensation mechanism to other well-known saturation compensation schemes. In particular, we design the decoupled anti-windup structure developed in [10] (we call the W-P anti-windup compensator for the seek of brevity) on top of the internal-model tracking controller, for the same tracking problem. As depicted in Figs. 19 and 20, although the commanded input of the W-P compensation scheme converges, it is still saturated severely and the system output cannot track the desired trajectory in presence of saturations. The RMS tracking error of the W-P compensator is $14.79 \mu \mathrm{m}$, while the robust anti-windup compensator is $0.44 \mu \mathrm{m}$. The comparison results illuminate that the proposed algorithm in the present paper significantly improves the saturation compensation capability.

\subsection{Robust performance}

Note that the proposed control architecture is designed with robustness against system uncertainties. As a matter of fact, the designed nano-manipulating system is required to carry various samples during the optical experiments, as illustrated in Fig. 1. Therefore, the robustness of the control scheme is very crucial for such applications. To this end, the circular tracking experiments are also conducted by mounting a weight of $50 \mathrm{~g}$ on the nano-stage as a load changing the system dynamics. From Fig. 21, it is straightforward that the proposed robust anti-windup tracking control architecture can handle saturations well and achieve good performance with a RMS tracking error of $0.67 \mu \mathrm{m}$. The results demonstrate decent robustness of the proposed control scheme under the influence of system uncertainties.

\section{Conclusion}

Aiming at supporting the ultramicroscopic imaging research by the SP confocal microscope, we proposed an XYZ nanomanipulator with large workspace and high mechanical bandwidth, as well as the robust anti-windup tracking control strategy for the nano-manipulating system in the present paper, such that asymptotic tracking can be achieved in the presence of saturation nonlinearity and model uncertainties. The stability and robustness conditions of the resulting closed loop system were analyzed and the controller design guidelines were provided in details with a trajectory tracking example. Real-time experiments demonstrated excellent tracking performance, saturation compensation capability and robust performance of 
the developed nano-manipulating system, which indicates good quality for high speed scanning in the SP confocal microscope. Future extensions along this line of research include the internal-model based anti-windup compensator with tracking capability and the discrete anti-windup tracking controller design.

\section{Acknowledgments}

We would like to thank the financial support from the NSFC under Grant Nos. 61327003 and 51775319, the National Key Research and Development Program of China under Grant No. 2017YFF0105903, and the Fundamental Research Funds of Shandong University under Grant No. 2015JC034.

\section{References}

[1] B. Zhang, S. Pechprasarn, J. Zhang, M.G. Somekh, Confocal surface plasmon microscopy with pupil function engineering, Opt. Express 20 (7) (2012) $7388-7397$.

[2] Y.K. Yong, B. Bhikkaji, S.O.R. Moheimani, Design, modeling, and FPAA-based control of a high-speed atomic force microscope nanopositioner, IEEE/ ASME Trans. Mechatron. 18 (3) (2013) 1060-1071.

[3] S. Devasia, E. Eleftheriou, S.O.R. Moheimani, A survey of control issues in nanopositioning, IEEE Trans. Control Syst. Technol. 15 (3) (2007) 802-823.

[4] G.Y. Gu, L.M. Zhu, C.Y. Su, H. Ding, S. Fatikow, Modeling and control of piezo-actuated nanopositioning stages: a survey, IEEE Trans. Autom. Sci. Eng. 13 (1) (2016) 313-332

[5] B.J. Kenton, K. Leang, Design and control of a three-axis serial-kinematic high-bandwidth nanopositioner, IEEE/ASME Trans. Mechatron. 17 (2) (2012) 356-369.

[6] G. Schitter, P.J. Thurner, P.K. Hansma, Design and input-shaping control of a novel scanner for high-speed atomic force microscopy, Mechatronics 18 (56) (2008) 282-288.

[7] S. Shao, M. Xu, S. Zhang, S. Xie, Stroke maximizing and high efficient hysteresis hybrid modeling for a rhombic piezoelectric actuator, Mech. Syst. Signal Process. 75 (2016) 631-647.

[8] J. Zheng, M. Fu, Saturation control of a piezoelectric actuator for fast settling-time performance, IEEE Trans. Control Syst. Technol. 21 (1) (2013) 220228.

[9] B. Zhou, G. Duan, Z. Lin, A parametric periodic Lyapunov equation with application in semi-global stabilization of discrete-time periodic systems subject to actuator saturation, Automatica 47 (2) (2011) 316-325.

[10] P.F. Weston, I. Postlethwaite, Linear conditioning for systems containing saturating actuators, Automatica 36 (9) (2000) $1347-1354$.

[11] G. Li, G. Herrmann, D.P. Stoten, J. Tu, M.C. Turner, A novel robust disturbance rejection anti-windup framework, Int. J. Robust Nonlinear Control 84 (1) (2011) 123-137.

[12] S. Gayadeen, S.R. Duncan, Discrete-time anti-windup compensation for synchrotron electron beam controllers with rate constrained actuators, Automatica 67 (2016) 224-232.

[13] X. Wu, Z. Lin, Dynamic anti-windup design in anticipation of actuator saturation, Int. J. Robust Nonlinear Control 24 (2) (2014) $295-312$.

[14] Z. Zhang, P. Yan, H. Jiang, P. Ye, A discrete time-varying internal model-based approach for high precision tracking of a multi-axis servo gantry, ISA Trans. 53 (5) (2014) 1695-1703.

[15] S. Necipoglu, S.A. Cebeci, C. Basdogan, Y.E. Hasb, L. Guvencc, Repetitive control of an XYZ piezo-stage for faster nano-scanning: numerical simulations and experiments, Mechatronics 21 (6) (2011) 1098-1107.

[16] Y. Zhang, P. Yan, Z. Zhang, Robust adaptive backstepping control for piezoelectric nano-manipulating systems, Mech. Syst. Signal Process. 83 (2017) 130-148.

[17] P. Liu, P. Yan, Z. Zhang, H. Özbay, Robust anti-windup compensation for high precision tracking of a piezoelectric nano-stage, IEEE Trans. Industr Electron. 63 (10) (2016) 6460-6470.

[18] P. Liu, P. Yan, Z. Zhang, T. Leng, Flexure-hinges guided nano-stage for precision manipulations: Design, modeling and control, Int. J. Precision Eng. Manufact. 16 (11) (2015) 2245-2254.

[19] L.L. Howell, Compliant Mechanisms, John Wiley \& Sons, 2001.

[20] V.M. Popov, Absolute stability of nonlinear systems of automatic control, Autom. Remote Control 22 (8) (1962) $857-875$.

[21] H.K. Khalil, Nonlinear Systems, Prentice Hall, New Jersey, 1996.

[22] B.A. Francis, A Course in $H_{\infty}$ Control Theory, Springer-Verlag, New York, 1987. 